\title{
The Spin-Boson model with a structured environment: A comparison of approaches
}

\author{
F.K. Wilhelm ${ }^{\mathrm{a}, *}$ S. Kleff ${ }^{\mathrm{a}}$ J. von Delft $^{\mathrm{a}}$ \\ a Sektion Physik and CeNS, Ludwig-Maximilians-Universität, Theresienstr. 37, \\ D-80333 München, Germany
}

\begin{abstract}
In the spin boson model, the properties of the oscillator bath are fully characterized by the spectral density of oscillators $J(\omega)$. We study the case when this function is of Breit-Wigner shape and has a sharp peak at a frequency $\Omega$ with width $\Gamma \ll \Omega$. We use a number of approaches such as the weak-coupling Bloch-Redfield equation, the non-interacting blip approximation (NIBA) and the flow-equation renormalization scheme. We show, that if $\Omega$ is much larger than the qubit energy scales, the dynamics corresponds to an Ohmic spin boson model with a strongly reduced tunnel splitting. We also show that the direction of the scaling of the tunnel splitting changes sign when the bare splitting crosses $\Omega$. We find good agreement between our analytical approximations and numerical results. We illuminate how and why different approaches to the model account for these features and discuss the interpretation of this model in the context of an application to quantum computation and read-out.
\end{abstract}

Key words: Spin boson model, quantum coputing, quantum measurement, cavity quantum electrodynamics

PACS: 03.65.Yz, 03.67.Lx, 05.40.-a, 85.25.Cp

\section{Introduction}

The subject of open-system quantum mechanics and the physics of the boundary between classical and quantum physics has been of strong interest since the early days of quantum theory. A paradigmatic standard model for the study of

\footnotetext{
* Corresponding author

Email address: wilhelm@theorie.physik. uni-muenchen.de (F.K. Wilhelm).
} 
open quantum systems is the spin boson model[1,2]: A single two-state system coupled to a bath of harmonic oscillators described by the Hamiltonian

$$
H=\frac{1}{2}\left(\epsilon \hat{\sigma}_{z}+\Delta \hat{\sigma}_{z}\right)+\frac{1}{2} \hat{\sigma}_{z} \sum_{i} c_{i} x_{i}+\frac{1}{2} \sum_{i}\left(\frac{p_{i}^{2}}{m}+m_{i} \omega_{i}^{2} x_{i}^{2}\right)+H_{0}
$$

Here, $H_{0}$ is a constant counter-term. The energy eigenvalues of the two state system alone are $\pm E / 2$ with $E=\sqrt{\epsilon^{2}+\Delta^{2}}$. The oscillator bath can model arbitrary Gaussian noise sources. It is fully characterized by a spectral function $J(\omega)$ which depends on the distribution of frequencies and couplings

$$
J(\omega)=\sum_{i} \frac{c_{i}^{2}}{2 m_{i} \omega_{i}^{2}} \delta\left(\omega-\omega_{i}\right)
$$

For a given physical system, e.g. a superconducting quantum bit coupled to a noisy electronic circuit, $J(\omega)$ can be obtained by analyzing either the effective friction [3] or noise [4] originating from the environment. It is useful to also introduce the semiclassical noise power $S(\omega)=J(\omega) \operatorname{coth}(\omega / 2 T)$.

Next to its long tradition in chemical physics, the physics of open quantum systems and in particular the spin boson model has gained recent practical importance in the field of quantum computation[5]. There, one is interested in obtaining long phase coherence times for the actual computation and long relaxation times for the readout. In a number of quantum computation realizations, the researcher has the option to taylor or engineer at least part of the properties of the quantum system and the dissipative environment under study [6], e.g. in the case of superconducting qubits coupled to their control and readout electronics[4]. In particular, environements with nontrivial internal dynamics, e.g. with resonances, can be realized and appear to be attractive $[4,7,8]$. Much is known about the physics of the spin boson model whose spectral density is a power law with an exponential cutoff $[1,2]$. Such spectral densities only contain the cutoff as an energy scale, which is typically assumed to be very high, leading to scale-free results.

Much less is known about structured environments. We are interested in a generic realization of this physics described by a spectral density containing a Breit-Wigner resonance

$$
J(\omega)=\alpha \omega \frac{\Omega^{4}}{\left(\omega^{2}-\Omega^{2}\right)^{2}+4 \omega^{2} \Gamma^{2}}
$$


in the underdamped case $\Gamma \ll \Omega$. In that case, we can expand

$$
J(\omega)=\frac{\alpha \Omega^{3}}{8 i \Gamma} \sum_{\sigma, \sigma^{\prime}= \pm 1} \frac{\sigma \sigma^{\prime}}{\omega-i \sigma^{\prime} \tilde{\Omega}-i \sigma \Gamma} \quad \tilde{\Omega}=\Omega-2 \Gamma^{2} / \Omega .
$$

Moreover, we will be able to profit from the analytical continuation of results with a Drude spectral density, noting that eq. 3 can be written as

$$
J(\omega)=\frac{\alpha \Omega^{3}}{4 i \Gamma} \sum_{\sigma= \pm 1} \frac{\sigma \omega}{\omega^{2}-(\sigma \tilde{\Omega}+i \Gamma)^{2}} .
$$

Note, that the shift of the resonance position from $\Omega$ towards $\tilde{\Omega}$ can be neglected in the underdamped case, except close to the resonance. Hence, we will henceforth only emphasize this shift in those cases, when it actually affects the results.

This type of spectral density is generically obtained by coupling the spin to a harmonic oscillator with eigenfrequency $\Omega$ which in turn is damped with a linear friction coeffcient $\Gamma / 2$. This friction is modeled quantum-mechanically by a bath of harmonic oscillators. Using a normal mode transformation, one can show that this is equivalent to our spin boson Hamiltonian with a structured bath [9]. More details are given in section 5. This model is realized in various physical systems such as chemical reactions involving biomolecules [9], atoms in cavities [10] or superconducting qubits coupled to resonators $[4,8,11,12,13,14]$. It corresponds to the nonlinear dimer model of polaron physics [15]. The case of no dissipation with restriction to a rotating wave approximation is known in quantum optics as the Jaynes-Cummings model[16]. Our notation corresponds to the one adopted in Ref. [7] and is slightly different to the one of Ref. [17].

The spin-boson model cannot be solved exactly and has been studied by a number of approaches. Some of them are largely numerical such as quantum Monte Carlo [18], real-time renormalization group [19], quasiadiabatic path integrals [20] flow equation renormalization [21] and numerical renormalization group [22], others are mainly analytical such as the noninteracting blip approximation (NIBA), a systematic weak damping approximation or exact Born approximation [23,24] or Bloch-Redfield [25,26,27]. A spectral density of the type eq. 3 poses a challenge to most of these approaches, since the spectral density in units of the frequency, $J(\omega) / \omega$, can be either very small (off-resonance) or large (on-resonance). In order to explore the phyiscs of this model and to obtain useful analytical information, these approximation schemes have to be applied within their range of validity and compared to numerical methods which are essentially nonperturbative in $J(\omega) / \omega$. Alternatively, one can treat the coupled TSS and oscillator system as multilevel quantum system and only 
the friction to the oscillator as a bath [28].

The plan of this paper is to analyze this model using the weak-coupling BlochRedfield theory and the nonperturbative NIBA and to compare the results to a full numerical study obtained in the flow equation scheme. We will very briefly introduce these methods and compare the dynamics of the reduced density matrix [ characterized through the expecation value $s_{z}(t)=\left\langle\hat{\sigma}_{z}\right\rangle(t)$ with, for definiteness, localized initial condition $s_{z}(0)=1$ ], effective reduced Hamiltonians, dephasing and coherence rates. Interpreteations of the results in terms of a superconducting quantum bit coupled to a resonant measurement circuit will be given.

\section{Bloch-Redfield}

The Bloch-Redfield-theory has originally been developped in the context of nuclear magnetic resonance[25]. It offers a systematic way to obtain a generalized master equation within the weak coupling Born approximation between system and bath with $J(\omega) / \omega$ as small parameter. It contains a subtle Markov approximation such that the resulting master equation is local in time; however, the main bath correlations relevant within the Born approximation are kept and they do lead to time-dependent rates for a driven system $[27,29]$. Bloch-Redfield has been shown to be numerically equivalent to a full nonMarkovian path integral technique for a rather generic choice of parameters [27]. Nevertheless, recent calculations at $T=0$ seem to indicate [24] that there may under certain circumstances be additional terms in the Born approximation, that are neglected in the Bloch-Redfield approach.

The natural starting point for the Bloch Redfield theory in the undriven case are the energy eigenstates of the spin-part of the Hamiltonian 1. In that "energy basis", the Bloch-Redfield equation can be written as (see e.g. ref. [2])

$$
\dot{\rho}_{n} m=-i \omega_{n m} \rho_{n m}+\sum_{k l} R_{n m k l} \rho_{k l}
$$

where all indices take the values + and - corresponding to the ground and excited state and $\omega_{n m}=\left(E_{n}-E_{m}\right) / \hbar$. The Redfield tensor has the form

$$
R_{n m k l}=\delta_{l m} \sum_{r} \Gamma_{n r r k}^{(+)}+\delta_{n k} \sum \Gamma_{l r r m}^{(-)}-\Gamma_{l m n k}^{(+)}-\Gamma_{l m n k}^{(-)}
$$

where we have introduced

$$
\Gamma_{l m n k}^{(+)}=\left(\sigma_{z}\right)_{l m}\left(\sigma_{z}\right)_{n k} \Gamma\left(\omega_{n k}\right) \quad \text { and } \quad \Gamma_{l m n k}^{(-)}=\left(\sigma_{z}\right)_{l m}\left(\sigma_{z}\right)_{n k} \Gamma^{*}\left(-\omega_{l m}\right)
$$


where $\left(\sigma_{z}\right)_{n k}$ are matrix elements of $\sigma_{z}$ in the energy basis, the ${ }^{*}$ denotes complex conjugation. The basic building block of the rates in the Redfield tensor is the rate $\Gamma$ which can be written as

$$
\Gamma(\delta \omega)=\frac{1}{2 \pi \hbar} \int_{0}^{\infty} d t e^{-i \delta \omega t} e^{-0^{+} t} \int_{0}^{\infty} d \omega J(\omega)[\cos \omega t \operatorname{coth}(\omega / 2 T)-i \sin \omega t] .
$$

The resulting dynamics displays exponential decay and reads

$$
s_{z}(t)=\frac{\epsilon^{2}}{E_{\text {eff }}^{2}}\left(e^{-\Gamma_{r} t}+\tanh \left(\frac{E_{\text {eff }}}{2 T}\right)\left(1-e^{-\Gamma_{r} t}\right)\right)+\frac{\Delta_{\text {eff }}^{2}}{E_{\text {eff }}^{2}} \cos \left(E_{\text {eff }} t\right) e^{-\Gamma_{\phi} t} .
$$

The quantities $\Delta_{\text {eff }}$ and $E_{\text {eff }}=\sqrt{\epsilon^{2}+\Delta_{\text {eff }}^{2}}$ can be associated with a renormalized Hamiltonian

$$
H_{\mathrm{eff}}=\frac{1}{2}\left(\epsilon \hat{\sigma}_{z}+\Delta_{\mathrm{eff}} \hat{\sigma}_{z}\right)
$$

The details of the shift of the tunnel splitting $\Delta \mapsto \Delta_{\text {eff }}$ will be discussed below.

The $\Gamma_{r}$ term in eq. 10 describes incoherent energy relaxation. It leads the system into thermal occupation of the renormalized Hamiltonian described below. The relaxation rate can be deduced from the Bloch-Redfield rates eqs. 9,7 and 8

$$
\begin{aligned}
\Gamma_{r} & =R_{----}+R_{++++}=\left(\sigma_{z}\right)_{-+}\left(\sigma_{z}\right)_{+-}(\Gamma(E)+\Gamma(-E)+\text { c.c. }) \\
& =\frac{\Delta^{2}}{2 E^{2}} S(E) .
\end{aligned}
$$

This result is easily understood in terms of the Born-approximation: In order to relax, the system has to exchange the energy corresponding to the energy splitting $E$ with the environment at once, using a single photon.

The last term in eq. 10 describes quantum coherent oscillations analogous to Larmor precession of a spin [30]. These are the hallmark of (macroscopic) quantum coherence in the spin boson system. Their decay rate can hence be identified with the dephasing rate and can, using eqs. 9, 7 and 8, be written as

$$
\begin{aligned}
\Gamma_{\phi} & =-\operatorname{Re} \Gamma_{-+-+}=\operatorname{Re}\left[2\left(\sigma_{z}\right)_{--}\left(\sigma_{z}\right)_{++} \Gamma(0)+\left(\sigma_{z}\right)_{-+}\left(\sigma_{z}\right)_{+-}\left(\Gamma(E)+\Gamma^{*}(-E)\right)\right] \\
& =\frac{\Gamma_{r}}{2}+\alpha T \frac{\epsilon^{2}}{\Delta^{2}} .
\end{aligned}
$$


Note, that on very general grounds [30] we have $2 \Gamma_{\phi} \geq \Gamma_{r}$. The extra factor of $1 / 2$ originates from the fact that there are in principle two dephasing channels corresponding to clockwise and counterclockwise Larmor precession. We are following here the standard NMR-motivated notation [30]; one could equivalently define $2 \Gamma_{\phi}$ as the true physical dephasing rate. The first term in eq. 13 is proportional to the relaxation rate eq. 12, which reflects that a relaxation process certainly also randomizes the phase information. The additional term involves $S(0)$, which in our case is $\propto T$. This contribution originates from "flipless" dephasing processes which randomize the phases while keeping energy constant, i.e. transitions from a state into itself.

The form of both rates eqs. 13 and 12 resembles the case of unstructured enviroments [23], even though the spectral density eq. 4 has singularities close to the real axis. The high relaxation rate at $E \simeq \Omega$ corresponds to resonant interaction between the qubit and the central environmental oscillator. When interpreting this result, one has to be aware, that the Born approximation involved is only valid for $\Gamma_{r, \phi} \ll E$, which, bounding $J(\omega) \leq J(\Omega)$, means $\alpha \Omega^{2}<\Gamma^{2}$. This a very rigorous constraint in the underdamped case, $\Gamma \ll \Omega$, which we are considering. Also physically, we do not expect this result to be consistent up to strong couplings, because the relatively weakly damped big oscillator is a highly coherent quantum system which mostly reversibly exchanges energy with the spin. However, since the Golden-Rule approximation in Bloch-Redfield only takes the long time limit, this reversible exchange cannot be seen in the Bloch-Redfield result. This can be understood from the order of limits prescribed by Bloch-Redfield and shown in the Appendix: The imaginary part of the energy is first sent to zero. Non-Markovian approximation schemes $[23,24]$ would at least take a self-consistent value and thus shift the $S(E)$ in eq. 12 into $\operatorname{Re} S\left(E+i \Gamma_{r}\right)$. Such shifts can be important in particular if $E \simeq \Omega$, when both predicted rates are very high. Results are summarized in figure 1 . We clearly see the peaked behavior at resonance and notice that the influence of the self-consistent solution is rather small even at rather extreme parameters. On the other hand, the self-consistent solution predicts lower rates as compaed to the non-self-consistent one, similar to the predictions of flow-equation studies [17].

As mentioned above, the environment not only causes dephasing and relaxation, it also renormalizes the tunnel splitting $\Delta$ (and with it the transition frequency), by dressing the two-state system with environmental degrees of freedom. This is similar to the physics of the Lamb shift or the Franck Condon effect and leads, in the nonperturbative regime, to the dissipative quantum phase transition $[1,31,32]$. In our case, the transition frequency is renormalized according to $E \rightarrow E-\operatorname{Im} R_{+-+-}$. If we look at the imaginary part of the generic rate, eq. A.2, $\Gamma^{\prime}(E)=\frac{1}{4 \pi \hbar} \int d \omega J(\omega) \mathcal{P} \frac{1}{\omega^{2}-E^{2}}[\operatorname{coth}(\beta \omega / 2) E-\omega]$ we observe a weight function $\mathcal{P}\left(\omega^{2}-E^{2}\right)^{-1}$ which changes sign at $\omega \simeq E$. Thus we can expect an upward renormalization of $E$ if most of the spectral weight of 


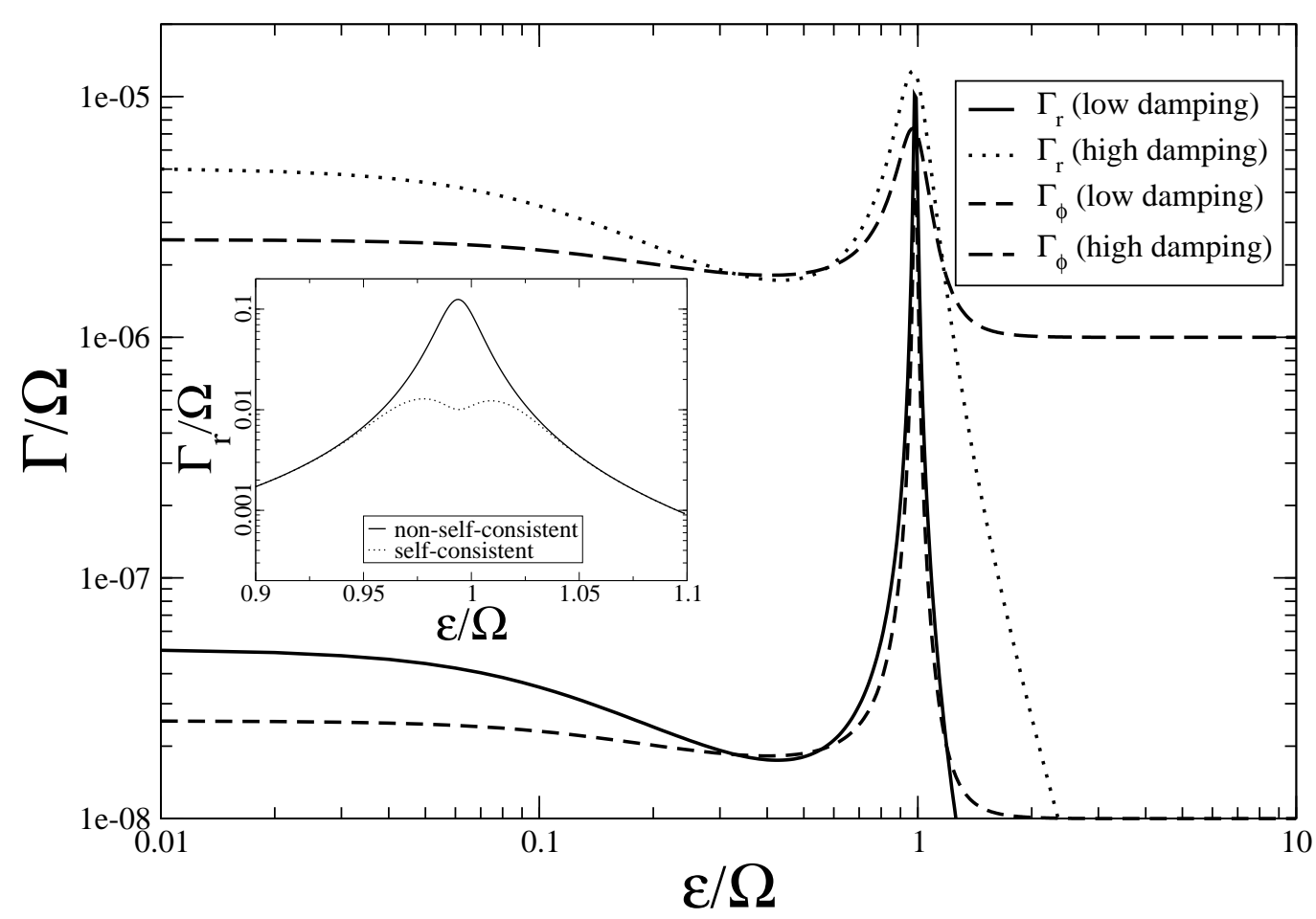

Fig. 1. Relaxation and dephasing rates as a function of energy bias predicted from Bloch-Redfield theory. Parameters are $\Delta=0.1 \Omega, T=0.01 \Omega$. For the low-damping plots we have chosen $\alpha=10^{-6}, \Gamma=10^{-2} \Omega$, for the high-damping plots we have chosen $\alpha=10^{-4}, \Gamma=10^{-4} \Omega$. The inset compares the self-consistent and non-self-consistent relaxation rate around the resonance for $\Delta=0.1 \Omega, T=0$, $\alpha=10^{-2}$ and $\Gamma=10^{-2} \Omega$.

$J(\omega)$ is above $E$ (corresponding to $E<\Omega$ ) whereas $E$ scales downward in the opposite case. Physically, this corresponds to level repulsion between the spin and the oscillators in the environment. The result also is consistent with usual second order perturbation theory for the energies. The sign changes happens at $E \simeq \Omega$, the point where most of the spectral weight is concentrated, thus we expect a rather sharp structure of the splitting $E_{\text {eff }}(\Omega)$. Note, that this sign change is not predicted for the usual spin-boson in the scaling limit, which can be studied by the well-known adiabatic renormalization approach[1,31]. In that case, $E_{\text {eff }}$ is always reduced. This is consistent with our findings, because in the scaling limit, the vast majority of the environmental oscillators have high frequency, much above the qubit splitting.

From the structure of the dephasing rate eq. 13 we can conclude that the last term in eq. A.3, which is even in energy, drops from the final result. Moreover, the remaining contribution to eq. A.3 vanishes as $E \rightarrow 0$. If we finally go to low temperatures, we can replace $p$ in eq. A.3 by an appropriate logarithm and find for the shift of the transition frequency

$$
\delta E=\frac{\Delta^{2}}{E^{2}} \frac{\alpha}{2 \pi} \frac{i \Omega^{2} E}{\Gamma} \sum_{\sigma} \frac{\sigma}{E^{2}-\left(\sigma \tilde{\Omega}^{2}+i \Gamma\right)^{2}} \log \left(\frac{\Gamma-i \sigma \tilde{\Omega}}{i E}\right) .
$$


In the underdamped limit we are working in, we can approximate the logarithm as $\log |\Omega / E|-i \sigma \pi / 2$ and split the result as $E_{\text {eff }}=E+\delta E, \delta E=$ $\delta E_{\Omega}+\delta E_{\text {res }}$. It contains a logarithmic contribution which resembles the scaling in the Ohmic case (with cutoff frequency $\Omega$ ),

$$
\delta E_{\Omega}=\frac{2}{\pi} \frac{\Delta^{2}}{E^{2}} J(E) \log \left|\frac{E}{\tilde{\Omega}}\right|
$$

This contribution changes sign from an upward shift at $\Omega<E$ to a downward shift at $\Omega>E$ as is expected from the general arguments above. The logarithmic divergence at low $E$ can be indicated as a precursor of a dissipative phase transition. The other contribution takes into account the enormous spectral weight of the resonance,

$$
\delta E_{\mathrm{res}}=\frac{\Delta^{2}}{E^{2}} J(E) \frac{E^{2}-\tilde{\Omega}^{2}}{\Omega \Gamma} .
$$

This contribution is of the order $\alpha / \Gamma$. It will be shown below, that terms of this kind persist even in the absence of damping of the external oscillator. It, too, undergoes the expected sign change. It is linear at low $E$ and hence does not contribute to a dissipative phase transition. It instead represents a substantial but finite renormalization. This is due to the fact, that for a dissipative phase transition, the environment has to get entangled with the spin down to arbitrarily small frequencies. These results are summarized in figure 2. As expected, we find in fig. 2, that the energy shift has a sharp structure around the sign change at $E \simeq \Omega$. At this point, the spin becomes strongly entangled with the central oscillator, hence the concept of "qubit energy splitting" is of limited applicability. This observation is consistent with the usual dressed atom approach of cavity quantum electrodynamics.

\section{NIBA}

So far, we have restricted ourselves to the Born approximation, i.e. to the lowest order in $J(\omega) / \omega$ and have otherwise kept the system general. We now turn to the noninteracting blip approximation (NIBA), which is nonperturbative in that parameter. It can be derived from evaluating the influence functional in a path-integral approach by assuming that the off-diagonal excursions ("blips") of the density matrix contributing to the path of the two state system are uncorrelated $[1,2]$. It is thus justified when $E \ll \Omega$, because then the bath is oscillating rapidly on the scale of the two-state system and the time-integrated bath correlation function quickly averages out, leading to weak damping on 

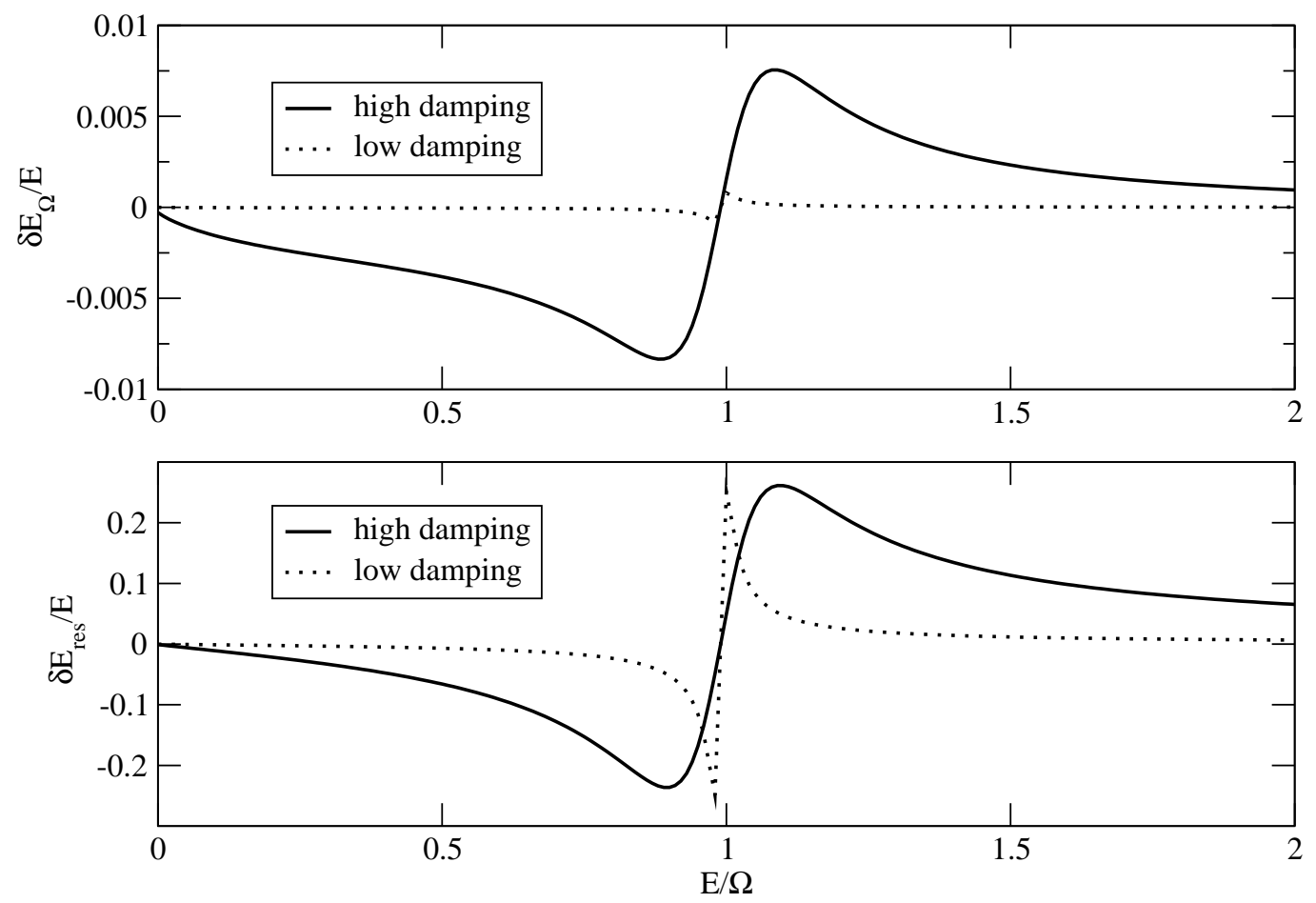

Fig. 2. Renormalization of the energy splitting at $T=0$ taken at the degeneracy point $\epsilon=0$, defined as positive if the splitting is decreased. Upper panel: Ohmic-like logarithmic contribution from eq. 15; lower panel: Contribution of the environmental resonance from eq. 16 (for discussion see text). Low damping: $\Gamma=0.01 \Omega, \alpha=10^{-4}$, high damping: $\Gamma=0.1 \Omega, \alpha=10^{-2}$.

longer time scales. Alternatively, the NIBA can be obtained by analyzing a the polaron-transfomed version of the spin-boson Hamiltonian.

NIBA is known to work well under these conditions at the degeneracy point $\epsilon=0$. At $\epsilon \neq 0$, the situation is more subtle. At $\epsilon \gg \Delta[1,2]$ the true dynamics is dominated by incoherent relaxation, which is again accurately predicted. This application of NIBA is closely related to the so-called $P(E)$ theory of Coulomb blockade $[2,33,34,35]$.

In this approach, the dynamics turns out to be governed by the Laplace transformed exponentiated correlation function

$$
P(\lambda)=\frac{\Delta^{2}}{2 \pi} \int_{0}^{\infty} e^{-\lambda t} e^{K(t)} d t
$$

where $K(t)$ is the twice integrated bath correlation function from eq. 9

$$
K(t)=\frac{1}{2 \pi} \int_{0}^{\infty} d t \frac{J(\omega)}{\omega^{2}}((\cos \omega t-1) \operatorname{coth}(\omega / 2 T)+i \sin \omega t)
$$


At the degeneracy point, the dynamics of the system in Laplace space is readily found from

$$
s_{z}(\lambda)=\int_{0}^{\infty} e^{-\lambda t} s_{z}(t)=\frac{1}{\lambda+\operatorname{Re} S(\lambda)}
$$

where $S(\lambda)=\left(P(\lambda)+P^{*}\left(\lambda^{*}\right)\right) / 2$. Far from the degeneracy point, we find incoherent relaxation

$$
s_{z}(t)=e^{-\Gamma_{r} t}\left[1-\tanh \left(\frac{\epsilon}{2 T}\right)\right]+\tanh \left(\frac{\epsilon}{2 T}\right) \quad \Gamma_{r}=2 \operatorname{Re} P(i \epsilon+0) .
$$

At $T=0$, we can use eq. 4 to evaluate $K(t)$ in closed form

$$
\begin{aligned}
K(t)= & \frac{\alpha \Omega}{8 \pi \Gamma i} \sum_{\sigma \sigma^{\prime}} \frac{\sigma^{\prime} \Omega-i \Gamma \sigma}{i \sigma^{\prime} \Gamma+\sigma \Omega}\left[e^{\left(i \sigma \Omega-\sigma^{\prime} \Gamma\right) t} \operatorname{Ei}\left(-\left(i \sigma \Omega-\sigma^{\prime} \Gamma\right) t\right)\right. \\
& \left.-\gamma-\log \left(-i\left(\sigma \Omega-\sigma^{\prime} \Gamma\right) t\right)\right]
\end{aligned}
$$

This is too compliated to allow a direct calculation of $P(\lambda)$ from eq. 17. At low energies, $E \ll \Omega$ we can concentrate on the long time limit of eq. 21 and find, keeping only lowest order terms in $\Gamma / \Omega$

$$
K_{\text {long }}(t)=-\frac{\alpha \Omega}{\Gamma}-2 \alpha[\log |\Omega t|+\gamma+i \pi / 2]
$$

where $\gamma$ is the Euler-Mascheroni-constant. This is a combination of a constant term of the order $\alpha / \Gamma$ and a logarithmic term which resembles the findings in the Ohmic case $[1,2]$. This is similar to what we observed in our Bloch-Redfield result for the scaling in eqs. 15 and 16 . From here, we find $P(\lambda)$ being

$$
P(\lambda)=e^{-\alpha \Omega / \Gamma} e^{-2 \gamma \alpha} e^{-i \alpha \pi} \frac{\Delta^{2}}{\lambda} \Gamma(1-2 \alpha)\left(\frac{\lambda}{\Omega}\right)^{2 \alpha}
$$

Off the degeneracy point, we can directly evaluate the relaxation rate from eq. 20 which reads

$$
\Gamma_{r}=\frac{\Delta^{2}}{|\epsilon|} e^{-\frac{\alpha \Omega}{\Gamma}} \frac{e^{-2 \gamma \alpha}}{\Gamma(2 \alpha)}\left(\frac{|\epsilon|}{\Omega}\right)^{2 \alpha} .
$$

This rate resembles to the Ohmic case $[33,2]$ but is reduced by an extra exponential prefactor $\exp (-\alpha \Omega / \Gamma)$, which again represents the contribution of the resonance and can be very small. Thus, we find te important result that 


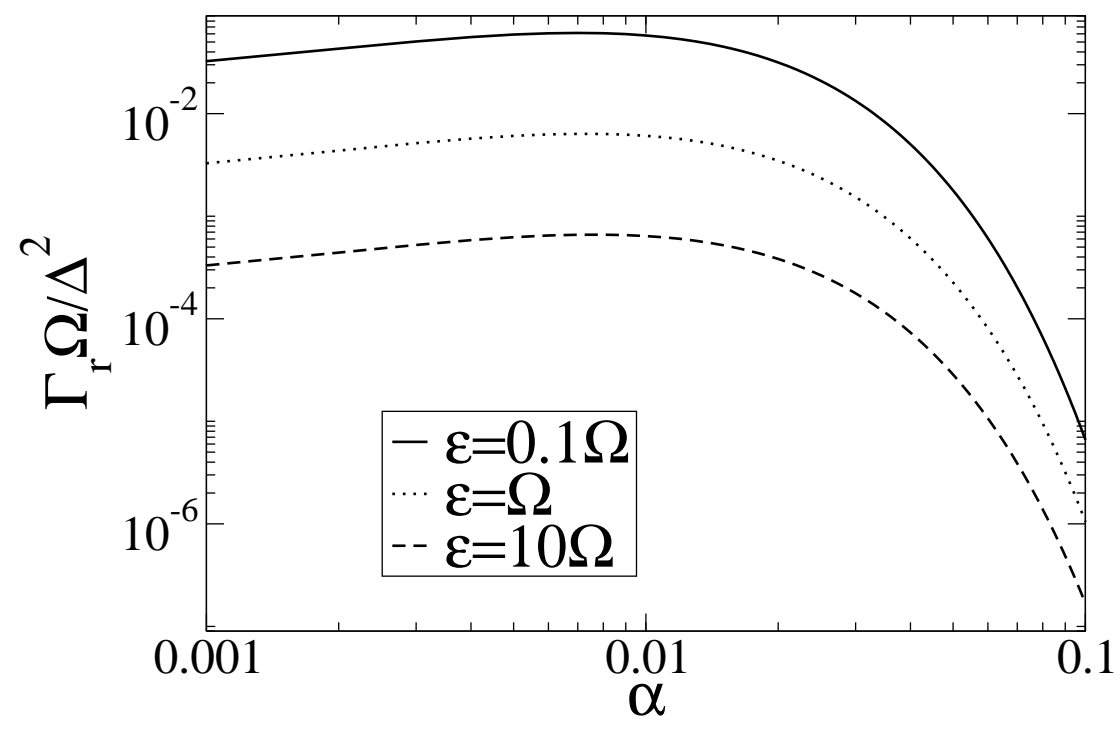

Fig. 3. Relaxation rates calculated from the NIBA eq. 24, in the long time approximation using $\Gamma=0.01 \Omega$. Note, that for small $\alpha$ values the rate first grows with growing $\alpha$, until the localization due to the resonance takes over and relaxation rates drop dramatically.

by desigining small $\alpha$ but appreciable $\alpha \Omega / \Gamma$, the incoherent relaxation rate of the spin can be reduced to extremely small values. A physical interpretation of this finding will be given later on. The predictions of equation 24 are shown in fig. 3. At the degeneracy point, at $\epsilon=0$, we find the Laplace transform of $s_{z}$ using eqs. 19 and 23. In analogy to the Ohmic case $[2,1]$ we obtain for the backtransform that

$$
s_{z}(t)=E_{2-2 \alpha}\left(-\left(\Delta_{\mathrm{eff}} t\right)^{2-2 \alpha}\right)
$$

where $E$ is the Mittag-Leffler function $[2,1,36]$ and

$$
\Delta_{\text {eff }}=\Delta\left(\frac{\Delta}{\Omega}\right)^{\alpha /(1-\alpha)}\left(e^{-\alpha \Omega / \Gamma} e^{-2 \gamma \alpha} \cos \pi \alpha \Gamma(1-2 \alpha)\right)^{1 /(2-2 \alpha)}
$$

is the renormalized tunnel splitting. Note that this is only valid at $\Delta \ll \Omega$ because we have taken the long time limit for $K(t)$. Consequently, it always predicts a downward renormalization. As in the Ohmic case, the dynamics show a crossover from decaying oscillations at low $\alpha$ to incoherent decay at $\alpha \geq$ $1 / 2$ at $\epsilon=0$. The renormalized tunneling frequency $\Delta_{\text {eff }}$ shows a combination of the usual Ohmic scaling behavior governed by $\alpha$, including a dissipative phase transition at $\alpha=1$, plus a very effective downscaling of $e^{-\alpha \Omega \pi / 8 \Gamma(1-\alpha)}$ governed by $\alpha / \Gamma$ only, which also occurs for an undamped resonance and is not present for the Ohmic case. This again captures the contribution of the resonance and reflects the behavior we have observed in eqs. 16 and fig. 2 to lowest order in $\alpha / \Gamma$. The dynamics is illustrated in figure 4 . We can observe, 

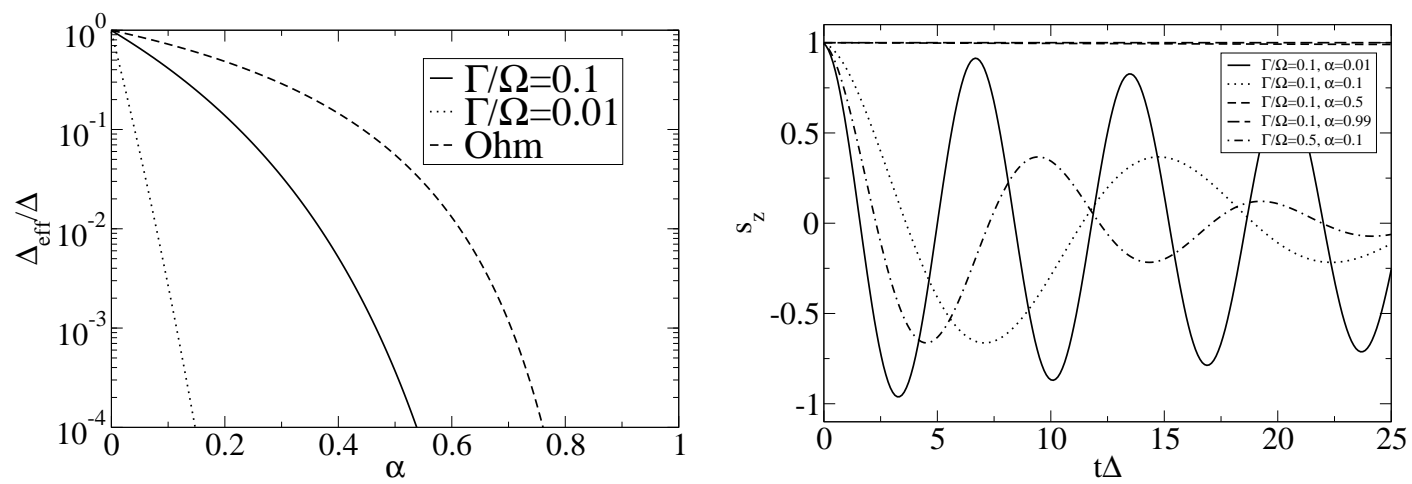

Fig. 4. Dynamical properties within the long time approximation of the NIBA at $\epsilon=0$. Left panel: Effective tunnel matrix element eq. 26 for $\Omega / \Delta=10$ for different values of the environment linewidth as a function of $\alpha$. Lower $\Gamma$ means more spectral weight and stronger downscaling of $\Delta_{\text {eff }}$. Right panel: Expectation value of $\sigma_{z}$, eq. 25 for different values of the damping parameters and $\Delta / \Omega=0.1$.

that the time evolution of the spin is almost brought to a standstill, in the sense of absence of both oscillations and decay, already at modest coupling constants.

\section{Comparison to flow equation results}

So far, we have studied our system using traditional methods for open quantum systems. In order to complement this work, we want to compare the above results with previous work [17], in which the same setup was studied with the flow-equation renormalization method [21], which originates from strongly correlated electron systems and very well suited for treating problems with several different energy scales. We will restrict ourselves to $\epsilon=0$. This method typically can be used to calculate spin-spin correlation functions in equilibrium such as $C(t)=\left\langle\sigma_{z}(t) \sigma_{z}(0)\right\rangle_{\text {eq. }}$. A typical example is shown in fig. 5. The Fourier-transformed correlation function $C(\omega)$ is peaked at several frequencies. The resonance around $\Delta_{\text {eff }}$ corresponds to coherent oscillations, its width can be identified with the dephasing rate. There can also be a resonance around $\Omega$ corresponding to oscillations of the oscillator leaving a trace on the qubit, but it hardly carries spectral weight. We have numerically solved the flow equations for small and moderate coupling strengths. More complete results are published elsewhere [17]. We see, that at $\Delta \ll \Omega, \Delta_{\text {eff }}$ is rescaled downwards similar to the NIBA, but with quantitative differences The BlochRedfield result produces the correct slope at small $\alpha$, see fig. 6 . Around $\Delta=\Omega$, the rescaling changes sign. Remarkably, Bloch-Redfield also predicts the slope above the sign change with good accuracy, see inset of fig. 6 , although this set of date is taken very close to resonance. Please note, that in the inset fig. 6 it is important to keep $\tilde{\Omega}$ in eq. 14 . 


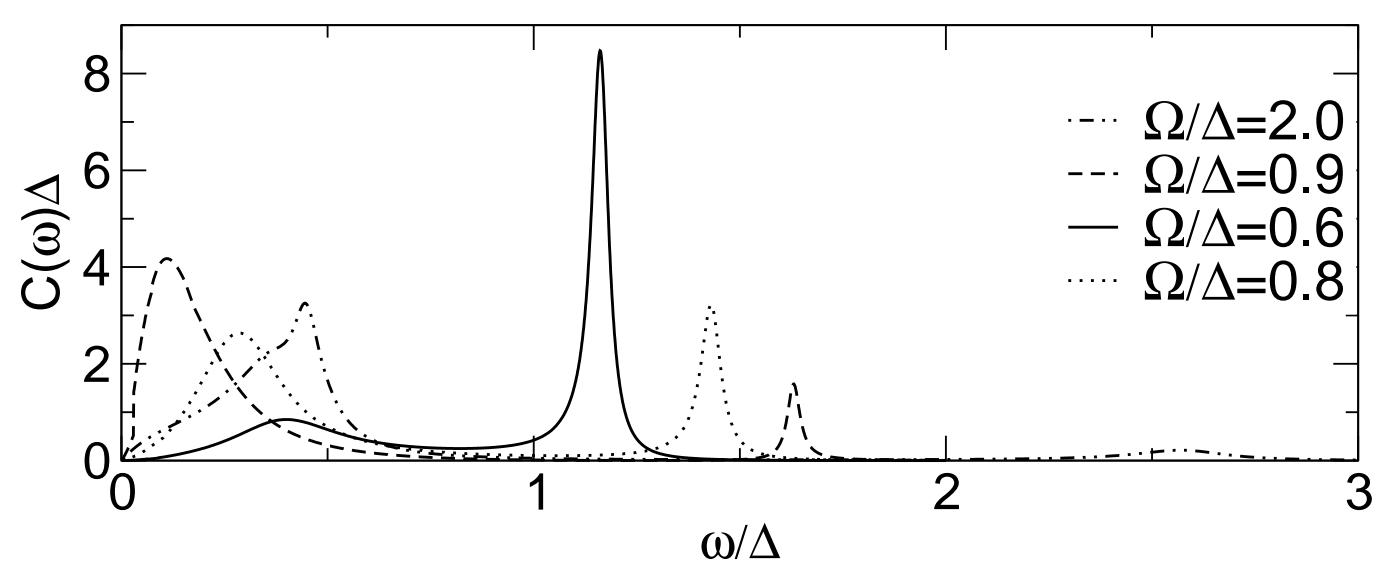

Fig. 5. Correlation function as evaluated from the flow-equation method using $\Omega / \Gamma=0.06$ and $\alpha=0.15$.

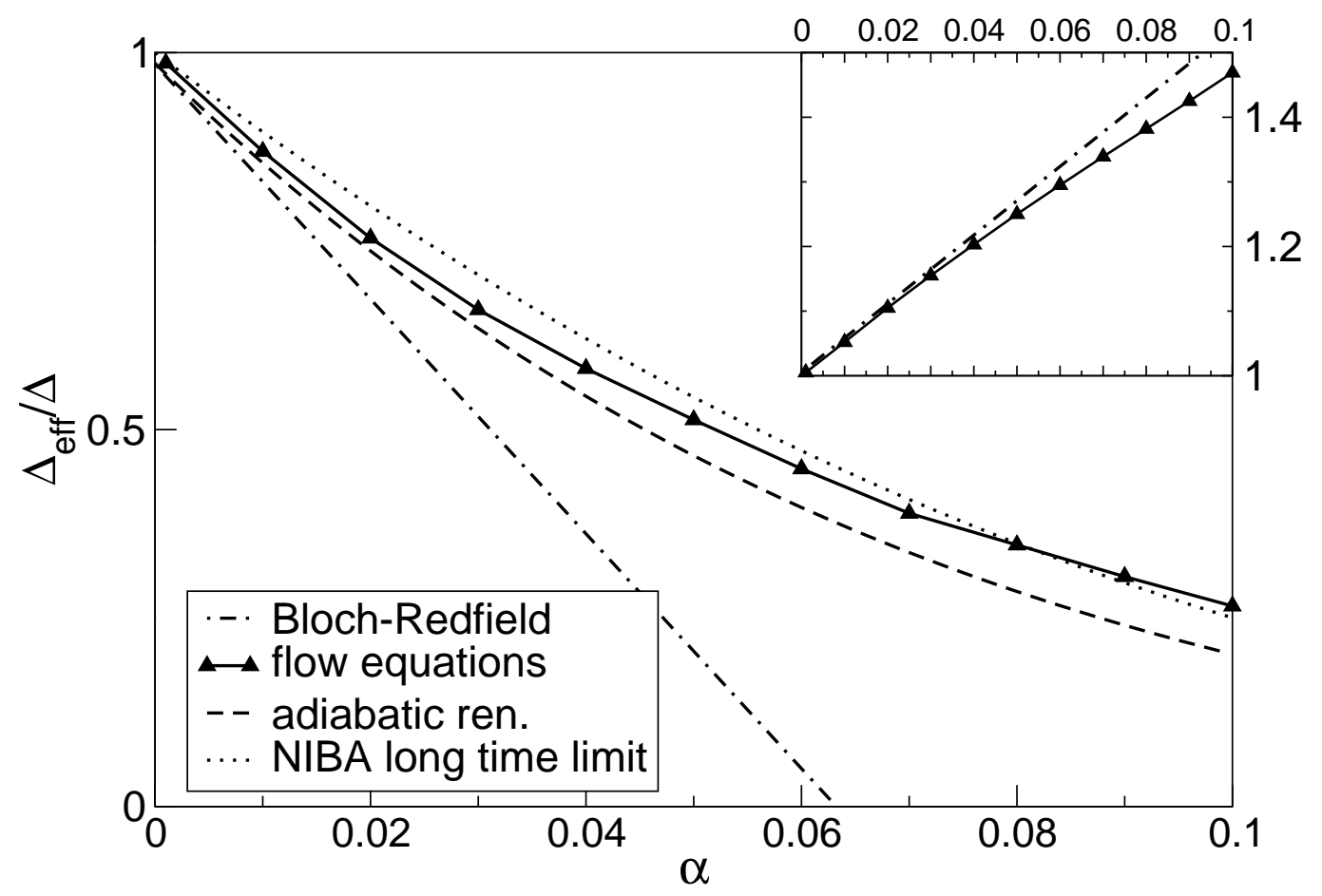

Fig. 6. Rescaling of the tunneling matrix element using different methods. Main plot: $\Gamma / \Omega=0.02 \pi, \Delta / \Omega=0.1$; inset: $\Gamma / \Omega=0.06 \pi, \Delta / \Omega=1.1$.

\section{Relation to quantum measurement and entanglement}

As already mentioned in the introduction, a straightforward way to implement this model with the spectral density eq. 3 is to couple the TSS to a single harmonic oscillator with resonance frequency $\Omega$, which is in turn damped by additional oscillators. This model has the Hamiltonian 


$$
\begin{aligned}
\hat{H}= & \frac{\epsilon}{2} \hat{\sigma}_{z}+\frac{\Delta}{2} \hat{\sigma}_{x}+\frac{\hat{P}^{2}}{2 M}+\frac{M}{2} \Omega^{2}\left(\hat{X}-q \hat{\sigma}_{z}\right)^{2} \\
& +\sum_{i}\left(\frac{\hat{p}_{i}^{2}}{2 m_{i}}+\frac{m_{i}}{2} \omega_{i}^{2}\left(\hat{x}_{i}-\left(\tilde{c}_{i} / m_{i} \omega_{i}^{2}\right) \hat{X}\right)^{2}\right) .
\end{aligned}
$$

The oscillator bath is characterized through an ohmic spectral density $\tilde{J}(\omega)=$ $\sum \frac{\pi \tilde{c}_{i}^{2}}{2 m_{i} \omega_{i}} \delta\left(\omega-\omega_{i}\right)=M \Gamma \omega$, where, $\Gamma / 2$ is the friction coefficient of the damped big oscillator. It was shown in [9], using a normal-mode decomposition, that this system is equivalent to the spin-boson Hamiltonian eq. 1 with spectral density eq. 3 , where $\alpha=2 M q^{2} \Gamma / \hbar$.

There are a number of realizations of such models. We would like to concentrate on a realization in superconducting quantum circuits: A flux quantum bit coupled to the plasma resonance of a DC-SQUID. This setup has been thoroughly analyzed in Refs. [3,4]. It has been shown that the spectral density of the flux noise indeed leads to eq. 3 and how the circuit parameters relate to the parameters of that function. Moreover, it has been shown that the coupling parameter $q$ actually can be tuned by the bias current through the SQUID. A representative circuit is shown in fig. 7. It is also shown there and discussed in Ref. [7], that a similar though less favorable realization can be found for charge quantum bits. We are mentioning this model, because it describes a detector of a quantum variable. Thus, we are going to interpret the findings of this paper in terms of quantum measurement theory. Other applications of resonantors coupled to superconducting qubits have been discussed in $[12,13,14]$ As a key result, we have found above within Bloch-Redfield as well as within NIBA, that at $\epsilon, \Delta \ll \Omega$, the system dynamics can be interpreted as an Ohmic spin boson model with a strongly downscaled tunneling matrix element. This can be understood in terms of the following model, which was introduced and discussed already in Ref. [7]. We start from the undamped case, $\Gamma=0$. the low-energy Hilbert space is spanned by $| \pm\rangle_{\text {eff }}=| \pm\rangle|L / R\rangle$ where $| \pm\rangle$ are the basis states of the qubit, $\sigma_{z}| \pm\rangle= \pm| \pm\rangle$ and $|L / R\rangle$ are coherent states of the harmonic oscillator centered around $X= \pm q$, see Fig. 8. So in a general low-energy state $|\psi\rangle=a|+\rangle_{\text {eff }}+b|-\rangle_{\text {eff }},|a|^{2}+|b|^{2}=1$, qubit and oscillator are entangled. In this low-energy basis, the Hamiltonian acquires the form of the renonrmalized spin part of the spin boson Hamiltonian eq. (11), with $\Delta_{\text {eff }}=\Delta\langle L \mid R\rangle=\Delta e^{-\eta}$, where $\eta=M \Omega q^{2} / \hbar$. This coincides with the result of eq. 26 in the limit of $\alpha \rightarrow 0$ but $\alpha / \Gamma=$ const. Under an appropriate choice of parameters, we can achieve $\eta>1$ and $\Delta_{\text {eff }} \ll \Delta$. Following the notion of Ref. [37], the degree of entanglement is equal to $1-e^{-2 \eta}=1-\left|\Delta_{\text {eff }} / \Delta\right|^{2}$, i.e. we can interpret strong separation of the preferred states of the external oscillator and strong renormalization,i.e. $\Delta_{\text {eff }} / \Delta \ll 1$ with strong entanglement. In terms of quantum measurement theory, the oscillator states are pointers onto the qubit states [38]. Chosing $\eta \gg 1$ corresponds to the condition of almost orthogonal pointer states in the environment, which has been identified as the condi- 


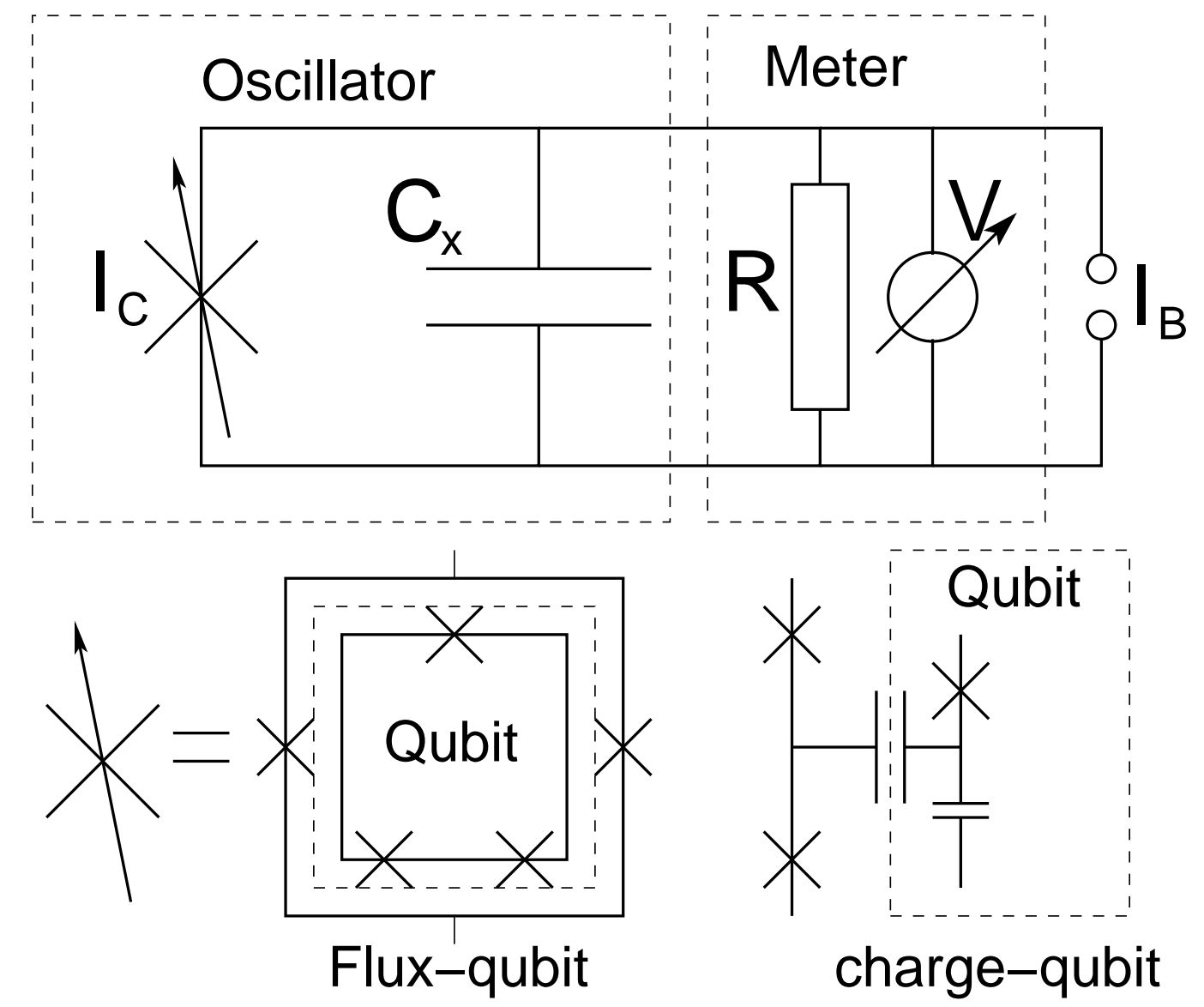

Fig. 7. Underdamped read-out devices for superconducting flux (left) and charge (right) quantum bits involving a tunable Josephson junction representing a SQUID or a superconducting SET.

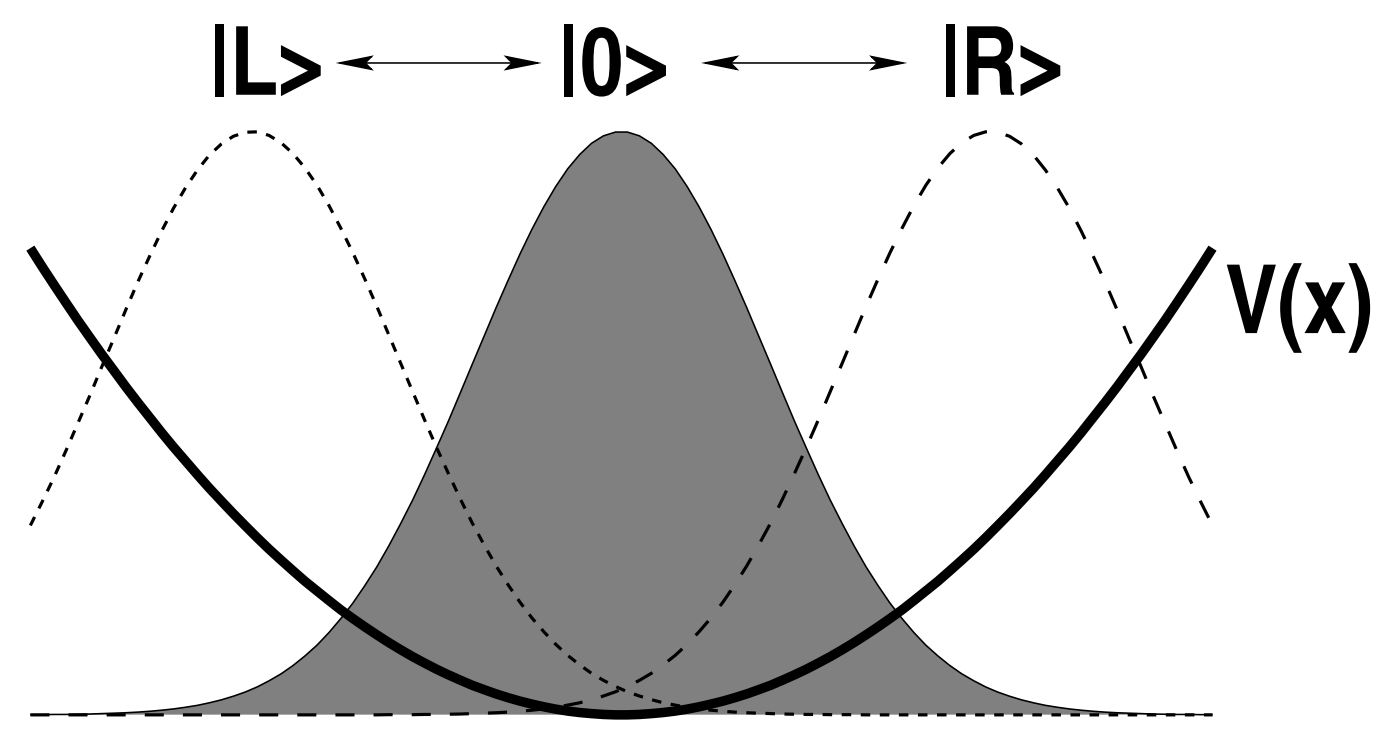

Fig. 8. Visualization of the ground state $|0\rangle$ and the coherent pointer-states $|L\rangle$ and $|R\rangle$ of the oscillator in the potential $V(x)$ 
tion for an ideal detector-dominated von-Neumann-measurement[38,39]. Such a measurement corresponds to the standard textbook quantum measurement: The preferred states into which superpositions are decohered are assumed to be Eigenstates of the measured observable regardless of the Hamiltonian of the qubit. In our case, eq. 27 describes coupling of the pointer degree of freedom to $\hat{\sigma}_{z}$ and hence measurement thereof. Rescaling $\Delta_{\text {eff }}$ asymptotically to zero means bringing the target states of the decoherence arbitrarily close to Eigenstates of $\hat{\sigma}_{z}$, thus realizing the aforementioned textbook assumption.

As it stands, the qubit just gets entangled with the pointers, but they are not read out. This can be done by coupling to the dissipative environment. As shown above, its influence corresponds to that of an Ohmic environment of strength $\alpha$. Taking $\alpha \ll 1$, this leads to dephasing and relaxation rates analogous to the Bloch Redfield results eqs. 12 and 13

$$
\Gamma_{\mathrm{r}}=\pi \alpha \frac{\Delta_{\text {eff }}^{2}}{E_{\text {eff }}} \operatorname{coth}\left(\frac{E_{\text {eff }}}{2 T}\right) \quad \Gamma_{\phi}=\frac{\Gamma_{\mathrm{r}}}{2}+2 \pi \alpha k_{\mathrm{B}} \frac{\epsilon^{2}}{E_{\mathrm{eff}}} T / \hbar .
$$

Note, that there may be nonexponential contributions to the dynamics as well $[24]$.

It is important to notice that in the strongly entangled case, $\Delta_{\text {eff }} \ll \Delta$, the relaxation rate, which describes the thermalization of the system independent from the initial state, is strongly reduced, whereas the dephasing rate, which describes the projection of a superposition into a mixture of the eigenstates $H_{\text {eff }}$ is hardly affected. This is a very favorable situation for a practical measurement: The information is quickly available, after $\tau_{\phi}=\Gamma_{\phi}^{-1}$ and is destroyed only after $\tau_{\mathrm{R}}=\Gamma_{\mathrm{R}}^{-1}$. This is not only convenient for experimental implementation but also guarantees high fidelity: The probability for reading out the correct result after the dephasing time is $P=e^{-\tau_{\phi} / \tau_{\mathrm{R}}}$ and thus close to unity. For completing the description of the measurement, one has to evaluate the resolution of the detector and the typical measurement times. This depends on details of the physical realization of interest and has been done in Ref. [7] for the superconducting setup.

\section{Summary and outlook}

We have studied the spin boson model with a structured bath using three different approaches: Bloch-Redfield, NIBA, and flow equation renormalization. We have arrived at a number of common features: If the peak in the spectral density is at frequencies much above the environmental resonance, the system is equivalent to a renormalized Ohmic spin boson model. This has been interpreted in terms of quantum measurement and the usefulness of this result for 
modeling quantum detectors has been outlined. We have furthermore shown that the tunneling matrix element of the spin part is renormalized downward if its initial value $\Delta$ is below the environmental resonance $\Omega$ and renormaized upward if it is above. We have compared this renormalization from all approaches and shown that they are in reasonable agreement within the scope of their applicability. In particular, our analytical results from NIBA and BlochRedfield reliably approximate the numerical results from flow equations.

This work would not have been possible without the extensive, illuminating and thorough work on the Spin Boson model by Prof. Uli Weiss. We would like to express our high regards and respect for him and his work and to congratulate him on his 60th birthday.

We would like to thank M. Grifoni and S. Kehrein for useful discussions. Work supported by the ARO under contract Nr. P-43385-PH-QC and through SFB 631.

\section{A Calculation of rates including poles of the spectral density}

We now want to outline how to calculate the rates eq. 9 We can interchange the order of integration and evaluate the time integral, which can be expanded into a delta function contribution and a Cauchy principal value. We can split $\Gamma(E)$ into real and imaginary part, $\Gamma^{\prime}(E)$ and $\Gamma^{\prime \prime}(E)$ and find

$$
\Gamma^{\prime}(E)=\frac{1}{8 \hbar} J(E)[\operatorname{coth}(\beta E / 2)-1]
$$

for the real part, which determines the decoherence and

$$
\Gamma^{\prime}(E)=\frac{1}{4 \pi \hbar} \int d \omega J(\omega) \mathcal{P} \frac{1}{\omega^{2}-E^{2}}[\operatorname{coth}(\beta \omega / 2) E-\omega]
$$

for the imaginary part, which controls the frequency shifts. The latter can be calculated by extending the integration contour to the complete real axis, applying the residue theorem and resumming the resulting Matsubara series. We end up with

$$
\Gamma={ }^{\prime \prime} \frac{\alpha}{2 \pi} \frac{\Omega^{2} E}{2 i \Gamma} \sum \frac{\sigma}{E^{2}-(\sigma \Omega+i \Gamma)^{2}}\left[p(\Gamma-i \sigma)-\operatorname{Rep}(i E)-\pi \frac{\Gamma-i \sigma \Omega}{E}\right]
$$

where $p(x)=\psi(1+\beta x / 2 \pi)+\psi(\beta x / 2 \pi)$ involves the digamma function $\psi$. 


\section{References}

[1] A.J. Leggett et al., Rev. Mod. Phys. 59, 1 (1987).

[2] U. Weiss, Quantum dissipative systems, 2nd ed., (World Scientific, Singapore, 1999).

[3] F.K. Wilhelm, M.J. Storcz, C.H. van der Wal, C.J.P.M. Harmans, and J.E. Mooij, to appear in Adv. Sol. St. Phys. Vol. 43; A.J. Leggett, Phys. Rev. B 30, 1208 (1984).

[4] C.H. van der Wal, F.K. Wilhelm, C.J.P.M. Harmans, and J.E. Mooij, Eur. Phys. J. B 31, 111-124 (2003).

[5] see e.g. M.A. Nielsen and I.L. Chuang, Quantum Computation and Quantum Information, (CUP, Cambridge, 2000).

[6] C.J. Myatt, B.E. King, QA. Tourchette, C.A. Sackett, D. Kielpinski, W.M. Itano, C. Monroe and D.J. Wineland, Nature 403, 269 (2000).

[7] F.K. Wilhelm, submitted to Phys. Rev. B

[8] J.M. Martinis, S. Nam, J. Aumentado, K.M. Lang and C. Urbina, Phys. Rev. B 67094510 (2003).

[9] A. Garg, J.N. Onuchic, and V. Ambegaokar, J. Chem. Phys. 83, 4491 (1985).

[10] M. Thorwart, L. Hartmann, I. Goychuk and P. Hänggi, J. Mod. Opt., 47, 2905 (2000).

[11] A.B. Zorin, Phys. Rev. Lett. 86, 3388 (2001).

[12] A. Blais, A. Maassen van der Brink, and A.M. Zagoskin, Phys. Rev. Lett. 90,127901 (2003).

[13] F. Marquardt and C. Bruder, Phys. Rev. B 63, 054514 (2001).

[14] F. Plastina and G. Falci, Phys. Rev. B 67, 224514 (2003).

[15] A.S. Davydov, Sov. Phys. Usp. 25, 898 (1982).

[16] E.T. Jaynes and F.W. Cummings, IEEE Proc. 51, 90 (1963).

[17] S. Kleff, S. Kehrein, and J. von Delft, submitted; cond-mat/0304177.

[18] R. Egger and U. Weiss, Z. Phys. B 89, 97 (1992).

[19] M. Keil and H. Schoeller, Phys. Rev. B 63, 180302(R) (2001).

[20] M. Thorwart, M. Grifoni, and P. Hänggi, Annals of Physics (New York) 293, $15(2001)$

[21] S.K. Kehrein and A. Mielke, Ann. Phys. (Leipzig) 6, 90 (1997).

[22] T.A. Costi and C. Kieffern Phys. Rev. Lett. 76, 1683 (1996). 
[23] M. Grifoni, E. Paladino, and U. Weiss, Eur. Phys. J. B 10, 719 (1999).

[24] D. Loss and D.P DiVincenzo, unpublished cond-mat/0304118.

[25] A.G. Redfield, Adv. Magn. Reson. 1, 1 (1965).

[26] P.N. Agyres and P.L. Kelley, Phys. Rev. 134, A 98 (1964).

[27] L. Hartmann, I. Goychuk, M. Grifoni and P. Hänggi, Phys. Rev. E 61, R4687 (2000).

[28] M. Thorwart et al., this volume.

[29] M.C. Goorden and F.K. Wilhelm, Phys. Rev. B 68, 012508 (2003).

[30] C.P. Slichter, "Principles of nuclear magnetic resonancs", 3rd ed., (Springer, New York, 1992).

[31] S. Chakravaty, Phys. Rev. Lett. 49, 681 (1982).

[32] A. Schmid, Phys. Rev. Lett. 51, 1506 (1983).

[33] G.-L. Ingold and Yu. V. Nazarov in H. Grabert and M. Devoret, eds., "Single charge tunneling" (NATO ASI Series, Plenum, 1992).

[34] G. Schön in T. Dittrich et al., "Quantum transport and dissipation" (WileyVCH, Weinheim, 1998).

[35] F.K. Wilhelm, G.T. Zimanyi, and G. Schön, Phys. Rev. Lett. 87, 136802 (2001).

[36] A. Erdelyi et al., Higher transcendental functions (McGraw Hill, N.Y., 1955).

[37] W. Dür, C. Simon, and J.I. Cirac, Phys. Rev. Lett. 89, 210402 (2002).

[38] W.H. Zurek, Phys. Rev. D 24, 1516 (1981); Prog. Theor. Phys. 89, 281 (1993).

[39] A. Peres Quantum Theory: Concepts and Methods, (Kluwer, Dordrecht, 1993). 